\title{
Asymptotics of solutions to the periodic problem for the nonlinear damped wave equation
}

Nakao Hayashi, Pavel I. Naumkin and Joel A. Rodriguez-Ceballos

\begin{abstract}
We study large time asymptotic behavior of solutions to the periodic problem for the nonlinear damped wave equation

$$
\left\{\begin{array}{l}
u_{t t}+2 \alpha u_{t}-\beta u_{x x}=-\lambda|u|^{\sigma} u, x \in \Omega, t>0, \\
u(0, x)=\phi(x), u_{t}(0, x)=\psi(x), x \in \Omega
\end{array}\right.
$$

where $\Omega=[-\pi, \pi], \alpha, \beta, \lambda, \sigma>0$. We prove that if the initial data $\phi \in \mathbf{H}^{1}$ and $\psi \in \mathbf{L}^{2}$, then there exists a unique solution $u(t, x) \in \mathbf{C}\left([0, \infty) ; \mathbf{H}^{1}\right)$ of the periodic problem which has the time decay estimates

$$
\|u(t)\|_{\mathbf{L}^{\infty}} \leq C\langle t\rangle^{-\frac{1}{\sigma}},\left\|\partial_{t} u(t)\right\|_{\mathbf{L}^{\infty}} \leq C\langle t\rangle^{-\frac{1}{\sigma}-\frac{1}{2}}
$$

for all $t>0$. Moreover under some additional conditions we find the asymptotic formulas for the solutions.
\end{abstract}

Mathematics Subject Classification (2000). 35L15, 35K20.

Keywords. Asymptotics of solutions, Periodic problem, Nonlinear damped wave equations.

\section{Introduction}

In this paper we study large time asymptotic behavior of solutions to the periodic problem for the nonlinear damped wave equation

$$
\left\{\begin{array}{l}
u_{t t}+2 \alpha u_{t}-\beta u_{x x}=-\lambda|u|^{\sigma} u, x \in \Omega, t>0, \\
u(0, x)=\phi(x), u_{t}(0, x)=\psi(x), x \in \Omega,
\end{array}\right.
$$

where $\Omega=[-\pi, \pi], \alpha, \beta, \lambda, \sigma>0$. We consider the solutions of Eq. (1.1), which satisfy the periodic boundary conditions $u(t, x)=u(t, 2 \pi+x)$ for all $x \in \mathbf{R}$ and $t>0$, with the $2 \pi$ - periodic initial data $\phi(x)$ and $\psi(x)$.

Recently much attention was drawn to the Cauchy problem for the nonlinear wave equations with different dissipative terms. The blow-up results were proved in [35] for the case of nonlinearity $|u|^{1+\sigma}$, with $\sigma<2$, when the initial data are such that $\int_{\mathbf{R}} \phi(x) d x>0$, and $\int_{\mathbf{R}} \psi(x) d x>0$. Blow-up results 
for the critical and subcritical cases $\sigma \leq 2$ were obtained in [20]. The global existence of solutions to the Cauchy problem for the nonlinear wave equations with nonlinearities $\pm|u|^{1+\sigma}$ or $\pm|u|^{\sigma} u$ for supercritical power $\sigma>2$ was proved in paper [35], where the large time decay estimates were obtained if the initial data are sufficiently small and have a compact support. The large time asymptotic behavior of solutions to the nonlinear damped wave equations for the supercritical case $\sigma>2$ was obtained in paper [13] in the framework of the usual $\mathbf{L}^{2}$-theory. When the initial data are in the usual Sobolev space $\partial^{\alpha} \phi \in \mathbf{L}^{1} \cap \mathbf{L}^{\infty},|\alpha| \leq 1, \psi \in \mathbf{L}^{1} \cap \mathbf{L}^{\infty}$, the Cauchy problem was considered in papers $[23,26,28]$. Applying the energy type estimates of [24], it was proved in paper [18] that solutions of the Cauchy problem in the supercritical case $\sigma>3$ with arbitrary initial data $\phi \in \mathbf{H}^{1} \cap \mathbf{L}^{1}, \psi \in \mathbf{L}^{2} \cap \mathbf{L}^{1}$ (i.e. without smallness assumption on the initial data) have the same large time asymptotics as that for the linear heat equation. The critical case $\sigma=2$ was considered in paper [12], where it was proved that the large time decay estimate of solutions of the Cauchy problem has an additional logarithmic correction. The large time asymptotics of solutions to the Cauchy problem with small initial data in the subcritical case $\sigma \in(0,2)$, when $\sigma$ is close to 2, was obtained in paper [14]. Note that similar behavior of solutions was discovered first for the nonlinear heat equation $u_{t}-u_{x x}-u^{1+\sigma}=0$ (see Ref. [40]). Sharp time decay estimates in $\mathbf{L}^{p}$ norm of solutions to the Cauchy problem in the subcritical case $\sigma \in(0,2)$ were obtained in paper [27], under the condition that the initial data decay exponentially at infinity without any restriction on the size. Asymptotic behavior of solutions to boundary value problems for abstract hyperbolic equations with dissipation (including the nonlinear damped wave equations) were studied in papers $[9,10,22,25,30-32]$ by using the weighted energy type estimates.

Large amount of literature is devoted to the investigation of periodic initial-boundary value problems. The asymptotic stability of stationary periodic solutions for the Fisher equation was proved in paper [7]. Periodic problem for the Hopf equation was studied in paper [15]. The asymptotic behavior for large time of solutions to the periodic problem for the Burgers equation with periodic force was found in paper [19]. In papers $[1,16,33]$ it was proved the existence of unique global solutions for the periodic problem to Korteweg-de Vries-Burgers equation and estimates of the large time decay rates of solutions are found. The approximate inertial manifolds for the damped forced Korteweg-de Vries-Burgers equation are constructed in Refs. [21,39]. Some time decay estimates of solutions of the Korteweg-de Vries-Burgers equation with periodic initial data with zero mean value were found in paper [1], where it was shown also that the asymptotic behavior is defined by the higher-order harmonics. In papers $[2,5,38]$ it is proved the existence of unique global solutions for the periodic problem to Landau-Ginzburg equation. Also time decay estimates of solutions in different norms were obtained and the blow up phenomena was studied for the case of large initial data. Exact periodic solutions for the Landau-Ginzburg equation were found in Ref. [21]. The approximate inertial manifolds for the Landau-Ginzburg equation were constructed in Ref. [29]. Existence of global solutions of derivative nonlinear Schrödinger equation 
was proved in Ref. [6]. Global existence and time decay estimates of solutions to the periodic problem for the Kuramoto-Sivashinsky equation were obtained in Ref. [11] and the attractors were studied in Ref. [8]. For the BenjaminBona-Mahony-Peregrine-Burgers equation, the existence of global solutions and decay estimates of energy were obtained in paper [37]. Large time behavior of solutions to the periodic problem for systems of conservation laws were studied in Refs. [4,34]. In paper [3] it was studied the existence and blow up of solutions to the periodic problem for the Camassa-Holm equation. The periodic problem for the Boussinesq equation was studies in paper [36]. The asymptotic formulas for solutions of the periodic problem to some nonlinear equations were found in Ref. [17].

As far as we know the large time asymptotic behavior of solutions to the periodic problem for the nonlinear damped wave Eq. (1.1) was not considered previously. Our aim is to prove large time asymptotic formulas for the solutions to the periodic problem for the nonlinear damped wave Eq. (1.1) without any restriction on the size of the initial data by applying the energy type estimates. We restrict our attention to the one dimensional case. However, we believe that the case of higher space dimensions also can be considered by our method. The study of the periodic problem is in many respects easier than the Cauchy problem and typically, the periodic results are exponential, whereas the problem on the line appears more delicate and often has algebraic decay rates (see Ref. [3]). Comparing with the Cauchy problem the asymptotic behavior of solutions to the periodic problem (1.1) is the same for all range of $\sigma>0$.

Before stating the results of this paper we give some notations. Denote the Sobolev space for the case of periodic functions

$$
\mathbf{H}^{s}=\left\{\phi \in \mathbf{D}^{\prime}:\|\phi\|_{\mathbf{H}^{s}}^{2}=\sum_{n=-\infty}^{\infty}\langle n\rangle^{2 s}\left|\widehat{\phi}_{n}\right|^{2}<\infty\right\}
$$

for any $s \in \mathbf{R}$. Here and below $\langle n\rangle=\sqrt{1+n^{2}}$ and $\widehat{\phi}_{n}=\frac{1}{2 \pi} \int_{\Omega} e^{-i n x} \phi(x) d x$ are the Fourier coefficients of the $2 \pi$-periodic function $\phi(x)$. For the functions with zero mean value we introduce the homogeneous Sobolev space $\dot{\mathbf{H}}^{s}=\left\{\phi \in \mathbf{H}^{s}: \widehat{\phi}_{0}=0\right\}$, so we have

$$
\|\phi\|_{\dot{\mathbf{H}}^{s}}^{2} \equiv \sum_{n=-\infty}^{\infty}\langle n\rangle^{2 s}\left|\widehat{\phi}_{n}\right|^{2}-\left|\widehat{\phi}_{0}\right|^{2}=\left\|\phi-\widehat{\phi}_{0}\right\|_{\mathbf{H}^{s}} .
$$

By $\mathbf{C}(\mathbf{I} ; \mathbf{B})$ we denote the space of continuous functions from a time interval I to the Banach space B. Different positive constants might be denoted by the same letter $C$.

We state our main results in this paper.

Theorem 1.1. Suppose that the initial data $\phi \in \mathbf{H}^{1}$ and $\psi \in \mathbf{L}^{2}$. Then there exists a unique solution $u(t, x) \in \mathbf{C}\left([0, \infty) ; \mathbf{H}^{1}\right)$ of the periodic problem (1.1) which obeys the time decay estimates 


$$
\|u(t)\|_{\mathbf{L}^{\infty}} \leq C\langle t\rangle^{-\frac{1}{\sigma}},\left\|\partial_{t} u(t)\right\|_{\mathbf{L}^{\infty}} \leq C\langle t\rangle^{-\frac{1}{\sigma}-\frac{1}{2}}
$$

for all $t \geq 0$.

By Theorem 1.1 the solution decays in time so we can consider the periodic problem (1.1) after sufficiently large time $T$ with sufficiently small initial data

$$
\|u(T)\|_{\mathbf{L}^{\infty}}+\left\|u_{t}(T)\right\|_{\mathbf{L}^{\infty}} \leq \varepsilon .
$$

So next we consider the periodic problem (1.1) with sufficiently small initial data.

Theorem 1.2. Suppose that the initial data $\phi \in \mathbf{H}^{1}, \psi \in \mathbf{L}^{2}$ are sufficiently small $\|\phi\|_{\dot{\mathbf{H}}^{1}}+\|\psi\|_{\mathbf{L}^{2}} \leq \varepsilon^{1+\frac{\sigma}{2}}$. Moreover, let the mean value $\widehat{\phi_{0}}=\varepsilon>0$. Then the asymptotics is true

$$
u(t, x)=A t^{-\frac{1}{\sigma}}+O\left(t^{-\frac{1}{\sigma}-\frac{1}{2}}\right)
$$

as $t \rightarrow \infty$ uniformly with respect to $x \in \Omega$, where $A=2 \alpha\left(\frac{2 \alpha}{\sigma \lambda}\right)^{\frac{1}{\sigma}}$.

We organize our paper as follows. In Sect. 2 we prepare some preliminary results in Lemmas 2.1-2.4. Then Sects. 3 and 4 are devoted to the proofs of Theorems 1.1 and 1.2, respectively.

\section{Preliminary estimates}

Consider first the linear periodic problem

$$
\left\{\begin{array}{l}
u_{t t}+2 \alpha u_{t}-\beta u_{x x}=f, x \in \Omega, t>0 \\
u(0, x)=\phi(x), u_{t}(0, x)=\psi(x), x \in \Omega,
\end{array}\right.
$$

where the force $f(t, x)$, and the initial data $\phi(x), \psi(x)$ are periodic with respect to the spatial variable $x: f(t, 2 \pi+x)=f(t, x), \phi(2 \pi+x)=\phi(x)$, $\psi(2 \pi+x)=\psi(x)$ for all $x \in \mathbf{R}, t>0$. Using the Fourier series we can formally represent the Green operator $\mathcal{G}(t)$ by

$$
\mathcal{G}(t) \psi=e^{-\alpha t} \sum_{n=-\infty}^{\infty} \widehat{\psi}_{n} e^{i x n} \frac{\sin \left(t \sqrt{\beta n^{2}-\alpha^{2}}\right)}{\sqrt{\beta n^{2}-\alpha^{2}}} .
$$

Therefore, the solution of the periodic problem can be written by the Duhamel formula

$$
u(t)=\widetilde{\mathcal{G}}(t) \phi+\mathcal{G}(t) \psi+\int_{0}^{t} \mathcal{G}(t-\tau) f(\tau) d \tau
$$

where $\widetilde{\mathcal{G}}(t)=\left(2 \alpha+\partial_{t}\right) \mathcal{G}(t)$.

We first collect some simple estimates for the solution of the linear periodic problem (2.1) in the Sobolev spaces $\mathbf{H}^{s}$ and $\dot{\mathbf{H}}^{s}$. Denote $\mu=\operatorname{Re}(\alpha-$ $\left.\sqrt{\alpha^{2}-\beta}\right)$. 
Lemma 2.1. Let $\phi \in \mathbf{H}^{s}, \psi \in \mathbf{H}^{s-1}$ and $f(t) \in \mathbf{H}^{s-1}$ with $s \geq 0$. Then the estimates are true

$$
\begin{aligned}
& \|\widetilde{\mathcal{G}}(t) \phi\|_{\mathbf{H}^{s}} \leq C\|\phi\|_{\mathbf{H}^{s}}, \quad\|\widetilde{\mathcal{G}}(t) \phi\|_{\dot{\mathbf{H}}^{s}} \leq C e^{-\mu t}\|\phi\|_{\dot{\mathbf{H}}^{s}}, \\
& \|\mathcal{G}(t) \psi\|_{\mathbf{H}^{s}} \leq C\|\psi\|_{\mathbf{H}^{s-1}},\|\mathcal{G}(t) \psi\|_{\dot{\mathbf{H}}^{s}} \leq C e^{-\mu t}\|\psi\|_{\dot{\mathbf{H}}^{s-1}}, \\
& \left\|\int_{0}^{t} \mathcal{G}(t-\tau) f(\tau) d \tau\right\|_{\mathbf{H}^{s}} \leq C \int_{0}^{t}\|f(\tau)\|_{\mathbf{H}^{s-1}} d \tau
\end{aligned}
$$

and

$$
\left\|\int_{0}^{t} \mathcal{G}(t-\tau) f(\tau) d \tau\right\|_{\dot{\mathbf{H}}^{s}} \leq C \int_{0}^{t} e^{-\mu(t-\tau)}\|f(\tau)\|_{\dot{\mathbf{H}}^{s-1}} d \tau
$$

for all $t>0$.

Proof. By the Parseval equality we find

$$
\|\widetilde{\mathcal{G}}(t) \phi\|_{\mathbf{H}^{s}}^{2}=\sum_{n=-\infty}^{\infty}\langle n\rangle^{2 s} e^{-2 \alpha t}\left|\cos \left(t \sqrt{\beta n^{2}-\alpha^{2}}\right)\right|^{2}\left|\widehat{\phi}_{n}\right|^{2} \leq C\|\phi\|_{\mathbf{H}^{s}}^{2}
$$

and

$$
\|\mathcal{G}(t) \psi\|_{\mathbf{H}^{s}}^{2}=\sum_{n=-\infty}^{\infty}\langle n\rangle^{2 s} e^{-2 \alpha t} \frac{\left|\sin \left(t \sqrt{\beta n^{2}-\alpha^{2}}\right)\right|^{2}}{\left|\beta n^{2}-\alpha^{2}\right|}\left|\widehat{\psi}_{n}\right|^{2} \leq C\|\psi\|_{\mathbf{H}^{s-1}}^{2} .
$$

In the same manner we obtain

$$
\|\widetilde{\mathcal{G}}(t) \phi\|_{\dot{\mathbf{H}}^{s}}^{2}=\sum_{|n| \geq 1}\langle n\rangle^{2 s} e^{-2 \alpha t}\left|\cos \left(t \sqrt{\beta n^{2}-\alpha^{2}}\right)\right|^{2}\left|\widehat{\phi}_{n}\right|^{2} \leq C e^{-2 \mu t}\|\phi\|_{\dot{\mathbf{H}}^{s}}^{2}
$$

and

$$
\|\mathcal{G}(t) \psi\|_{\dot{\mathbf{H}}^{s}}^{2}=\sum_{|n| \geq 1}\langle n\rangle^{2 s} e^{-2 \alpha t} \frac{\left|\sin \left(t \sqrt{\beta n^{2}-\alpha^{2}}\right)\right|^{2}}{\left|\beta n^{2}-\alpha^{2}\right|}\left|\widehat{\psi}_{n}\right|^{2} \leq C e^{-2 \mu t}\|\psi\|_{\dot{\mathbf{H}}^{s-1}}^{2} .
$$

Applying the above estimates we get

$$
\begin{aligned}
\left\|\int_{0}^{t} \mathcal{G}(t-\tau) f(\tau) d \tau\right\|_{\mathbf{H}^{s}} & \leq \int_{0}^{t}\|\mathcal{G}(t-\tau) f(\tau)\|_{\mathbf{H}^{s}} d \tau \\
& \leq C \int_{0}^{t}\|f(\tau)\|_{\mathbf{H}^{s-1}} d \tau
\end{aligned}
$$

and

$$
\begin{aligned}
\left\|\int_{0}^{t} \mathcal{G}(t-\tau) f(\tau) d \tau\right\|_{\dot{\mathbf{H}}^{s}} & \leq \int_{0}^{t}\|\mathcal{G}(t-\tau) f(\tau)\|_{\dot{\mathbf{H}}^{s}} d \tau \\
& \leq C \int_{0}^{t} e^{-\mu(t-\tau)}\|f(\tau)\|_{\dot{\mathbf{H}}^{s-1}} d \tau .
\end{aligned}
$$

Lemma 2.1 is proved. 
We next prove the local in time existence of the solutions to the periodic problem (1.1).

Lemma 2.2. Suppose that the initial data $\phi \in \mathbf{H}^{s}$ and $\psi \in \mathbf{H}^{s-1}$ with $1 \geq$ $s>\frac{1}{2}$ and $\sigma>0$. Then for some time $T>0$ there exists a unique solution $u(t, x) \in \mathbf{C}\left([0, T] ; \mathbf{H}^{s}\right)$ of the periodic problem (1.1).

Proof. Denote $\mathcal{N}(u)=-\lambda|u|^{\sigma} u$. By virtue of the Green operator $\mathcal{G}(t)$ of the linear periodic problem (2.1) we write the nonlinear periodic problem (1.1) in the form of the integral equation

$$
u(t)=\widetilde{\mathcal{G}}(t) \phi+\mathcal{G}(t) \psi+\int_{0}^{t} \mathcal{G}(t-\tau) \mathcal{N}(u)(\tau) d \tau .
$$

We solve Eq. (2.2) by the contraction mapping principle. We define the transformation

$$
\mathcal{A} v(t)=\widetilde{\mathcal{G}}(t) \phi+\mathcal{G}(t) \psi+\int_{0}^{t} \mathcal{G}(t-\tau) \mathcal{N}(v)(\tau) d \tau
$$

in the space $\mathbf{C}\left([0, T] ; \mathbf{H}^{s}\right)$, where the value $T>0$ will be chosen below. By the inequality $|\mathcal{N}(u)-\mathcal{N}(v)| \leq C\left(|u|^{\sigma}+|v|^{\sigma}\right)|u-v|$ we have

$$
\|\mathcal{N}(u)-\mathcal{N}(v)\|_{\mathbf{H}^{s-1}} \leq C\left(\|u\|_{\mathbf{H}^{s}}^{\sigma}+\|v\|_{\mathbf{H}^{s}}^{\sigma}\right)\|u-v\|_{\mathbf{H}^{s}}
$$

for any functions $u, v \in \mathbf{H}^{s}$ with $s>\frac{1}{2}$. By virtue of Lemma 2.1 we get

$$
\begin{aligned}
& \sup _{t \in[0, T]}\|\mathcal{A} v(t)\|_{\mathbf{H}^{s}} \\
& \leq \sup _{t \in[0, T]}\left(\|\widetilde{\mathcal{G}}(t) \phi\|_{\mathbf{H}^{s}}+\|\mathcal{G}(t) \psi\|_{\mathbf{H}^{s}}+\left\|\int_{0}^{t} \mathcal{G}(t-\tau) \mathcal{N}(v)(\tau) d \tau\right\|_{\mathbf{H}^{s}}\right) \\
& \leq C\|\phi\|_{\mathbf{H}^{s}}+C\|\psi\|_{\mathbf{H}^{s-1}}+C T \sup _{t \in[0, T]}\|\mathcal{N}(v)(t)\|_{\mathbf{H}^{s-1}} \\
& \leq C\|\phi\|_{\mathbf{H}^{s}}+C\|\psi\|_{\mathbf{H}^{s-1}}+C T \sup _{t \in[0, T]}\|v\|_{\mathbf{H}^{s}}^{\sigma+1} .
\end{aligned}
$$

Hence, we see that there exists a sufficiently small time $T$ which depends on the norm of the initial data $\|\phi\|_{\mathbf{H}^{s}}+\|\psi\|_{\mathbf{H}^{s-1}}$ such that $\sup _{t \in[0, T]}\|\mathcal{A} v(t)\|_{\mathbf{H}^{s}} \leq$ $2 C\left(\|\phi\|_{\mathbf{H}^{s}}+\|\psi\|_{\mathbf{H}^{s-1}}\right)$. In the same manner we estimate the difference

$$
\begin{aligned}
& \sup _{t \in[0, T]}\|\mathcal{A} u(t)-\mathcal{A} v(t)\|_{\mathbf{H}^{s}} \\
& \quad \leq \sup _{t \in[0, T]}\left\|\int_{0}^{t} \mathcal{G}(t-\tau)(\mathcal{N}(u)-\mathcal{N}(v))(\tau) d \tau\right\|_{\mathbf{H}^{s}} \\
& \quad \leq C T \sup _{t \in[0, T]}\|u(\tau)-v(\tau)\|_{\mathbf{H}^{s}}\left(\|u(\tau)\|_{\mathbf{H}^{s}}^{\sigma}+\|v(\tau)\|_{\mathbf{H}^{s}}^{\sigma}\right) \\
& \quad \leq \frac{1}{2} \sup _{t \in[0, T]}\|u(\tau)-v(\tau)\|_{\mathbf{H}^{s}}
\end{aligned}
$$

if $T>0$ is sufficiently small. Therefore, the transformation $\mathcal{A}$ is the contraction mapping in the closed ball of a radius $2 C\left(\|\phi\|_{\mathbf{H}^{s}}+\|\psi\|_{\mathbf{H}^{s-1}}\right)$ in the space 
$\mathbf{C}\left([0, T] ; \mathbf{H}^{s}\right)$. Hence, there exists a unique solution $u(t, x) \in \mathbf{C}\left([0, T] ; \mathbf{H}^{s}\right)$ of the periodic problem (1.1). Lemma 2.2 is proved.

Now we prove the global existence of solutions.

Lemma 2.3. Suppose that the initial data $\phi \in \mathbf{H}^{1}$ and $\psi \in \mathbf{L}^{2}$. Then there exists a unique global in time solution $u(t, x) \in \mathbf{C}\left([0, \infty) ; \mathbf{H}^{1}\right)$ of the periodic problem (1.1). Moreover for any $\varepsilon>0$ there exists a time $T$ such that

$$
\|u(t)\|_{\mathbf{H}^{1}}+\left\|u_{t}(t)\right\|_{\mathbf{L}^{2}} \leq \varepsilon
$$

for all $t \geq T$.

Proof. We use the energy type estimates. Let $u$ be a solution constructed in Lemma 2.2. We multiply Eq. (1.1) by $2 u_{t}+\alpha u$. Then integrating over $\Omega$ we get

$$
\begin{aligned}
0= & \int_{\Omega}\left(2 u_{t} u_{t t}+4 \alpha u_{t}^{2}-2 \beta u_{t} u_{x x}+2 \lambda|u|^{\sigma} u u_{t}\right. \\
& \left.+\alpha u u_{t t}+2 \alpha^{2} u u_{t}-\beta \alpha u u_{x x}+\lambda \alpha|u|^{\sigma+2}\right) d x .
\end{aligned}
$$

Then integrating by parts with respect to $x$ and taking into account the periodic boundary conditions we find

$$
\frac{d E}{d t}+H=0
$$

where the energy

$$
E=\int_{\Omega}\left(u_{t}^{2}+\alpha u u_{t}+\alpha^{2} u^{2}+\beta u_{x}^{2}+\frac{2 \lambda}{\sigma+2}|u|^{\sigma+2}\right) d x
$$

and

$$
H=\int_{\Omega}\left(3 \alpha u_{t}^{2}+\frac{\beta}{2} \alpha u_{x}^{2}+\lambda \alpha|u|^{\sigma+2}\right) d x .
$$

Note that $H \geq 0$. Thus, we obtain the inequality $\frac{d E}{d t} \leq 0$. Integrating we get $E(t) \leq E(0)$. Which yields the estimate for the solution

$$
\|u(t)\|_{\mathbf{H}^{1}}+\left\|u_{t}(t)\right\|_{\mathbf{L}^{2}}+\|u(t)\|_{\mathbf{L}^{\sigma+2}} \leq C
$$

for all $t>0$. This a-priory estimate by a standard continuation argument implies the existence of the global in time solution $u(t, x) \in \mathbf{C}\left([0, \infty) ; \mathbf{H}^{1}\right)$ of the periodic problem (1.1). Also we have the estimate

$$
\int_{0}^{\infty} H(t) d t \leq C
$$

This implies that for any $\varepsilon>0$ there exists a time $T$ such that $\|u(T)\|_{\mathbf{H}^{1}}+$ $\left\|u_{t}(T)\right\|_{\mathbf{L}^{2}} \leq \varepsilon$. Since the energy $E(t)$ decays in time we have

$$
\|u(t)\|_{\mathbf{H}^{1}}+\left\|u_{t}(t)\right\|_{\mathbf{L}^{2}} \leq \varepsilon
$$

for all $t \geq T$. Lemma 2.3 is proved. 
By Lemma 2.3 we can reduce the study of the asymptotics of solutions to the case of small initial data.

We next prove the following auxiliary result. Consider the ordinary differential equation

$$
y^{\prime \prime}+2 \alpha y^{\prime}=-\lambda|y|^{\sigma} y+\varphi(t) .
$$

Lemma 2.4. Let $\varphi \in \mathbf{C}([0, \infty))$ and $|\varphi(t)| \leq C\langle t\rangle^{-\frac{1}{\sigma}-\frac{3}{2}}$. Suppose that $|y(t)|+$ $\left|y^{\prime}(t)\right| \leq \varepsilon$ for all $t \geq 0$, where $\varepsilon>0$ is small enough. Then the solutions of Eq. (2.3) satisfy the estimates

$$
\langle t\rangle^{\frac{1}{\sigma}}|y(t)|+\langle t\rangle^{\frac{1}{\sigma}+\frac{1}{2}}\left|y^{\prime}(t)\right| \leq C .
$$

Proof. We multiply (2.3) by $2 y^{\prime}+|y|^{\sigma} y$, then

$$
\frac{d}{d t} E(t)=-\left(4 \alpha-(1+\sigma)|y|^{\sigma}\right)\left(y^{\prime}\right)^{2}-\lambda|y|^{2+2 \sigma}+\left(2 y^{\prime}+|y|^{\sigma} y\right) \varphi(t) .
$$

where we denote $E(t)=\left(y^{\prime}\right)^{2}+|y|^{\sigma} y y^{\prime}+\frac{2(\alpha+\lambda)}{2+\sigma}|y|^{2+\sigma}$. Note that $\left.|| y\right|^{\sigma} y y^{\prime} \mid \leq$ $\frac{1}{2}|y|^{2+2 \sigma}+\frac{1}{2}\left(y^{\prime}\right)^{2}$, hence by the condition $|y| \leq \varepsilon$, we have the estimate

$$
E(t) \geq \frac{1}{2}\left(y^{\prime}\right)^{2}+\frac{\alpha+\lambda}{2+\sigma}|y|^{2+\sigma} .
$$

Also we get the estimates

$$
\begin{aligned}
- & \left(4 \alpha-(1+\sigma)|y|^{\sigma}\right)\left(y^{\prime}\right)^{2}-\lambda|y|^{2+2 \sigma}+\left(2 y^{\prime}+|y|^{\sigma} y\right) \varphi(t) \\
& \leq-2 \alpha\left(y^{\prime}\right)^{2}-\frac{\lambda}{2}|y|^{2+2 \sigma}+C \varphi^{2}(t) \leq-C E^{\frac{2+2 \sigma}{2+\sigma}}(t)+C \varphi^{2}(t) .
\end{aligned}
$$

Thus, we find

$$
\frac{d}{d t} E(t) \leq-C E^{\frac{2+2 \sigma}{2+\sigma}}(t)+C \varphi^{2}(t) .
$$

Integrating this inequality yields the estimate $E(t) \leq C\langle t\rangle^{-1-\frac{2}{\sigma}}$. This implies the time decay estimate of the lemma. Lemma 2.4 is proved.

Now we study the case of small initial data.

Lemma 2.5. Let $\varphi \in \mathbf{C}([0, \infty))$ and $|\varphi(t)| \leq C \varepsilon^{1+\sigma}\langle t\rangle^{-\frac{1}{\sigma}-\frac{3}{2}}$. Suppose that $|y(0)| \leq \varepsilon$ and $\left|y^{\prime}(0)\right| \leq \varepsilon^{1+\frac{\sigma}{2}}$, where $\varepsilon>0$ is small enough. Then the solutions of Eq. (2.3) satisfy the estimates

$$
|y(t)| \leq C \varepsilon\langle t\rangle^{-\frac{1}{\sigma}},\left|y^{\prime}(t)\right| \leq C \varepsilon^{1+\frac{\sigma}{2}}\langle t\rangle^{-\frac{1}{\sigma}-\frac{1}{2}} .
$$

Proof. Again we arrive to (2.5)

$$
\frac{d}{d t} E(t) \leq-C E^{\frac{2+2 \sigma}{2+\sigma}}(t)+C \varepsilon^{2+2 \sigma}\langle t\rangle^{-\frac{2}{\sigma}-3} .
$$

Integrating (2.7) yields the estimate

$$
\left|y^{\prime}\right|^{2}+|y|^{2+\sigma} \leq C E(t) \leq C \varepsilon^{2+\sigma}\langle t\rangle^{-1-\frac{2}{\sigma}} .
$$

This implies the time decay estimate (2.6). Lemma 2.5 is proved. 


\section{Proof of Theorem 1.1}

Global existence of a unique solution $u(t, x) \in \mathbf{C}\left([0, \infty) ; \mathbf{H}^{1}\right)$ of the periodic problem (1.1) follows from Lemma 2.3. Also after some time $T$ we have the estimate

$$
\|u(t)\|_{\mathbf{H}^{1}}+\left\|u_{t}(t)\right\|_{\mathbf{L}^{2}} \leq \varepsilon
$$

for all $t \geq T$. To prove the asymptotic formula for the solution we consider the periodic problem (1.1) with the initial time $T$. By the shift $t^{\prime}=t-T$ we again arrive to the periodic problem (1.1) with the initial time at the origin and some small initial data $\phi \in \mathbf{H}^{1}$ and $\psi \in \mathbf{L}^{2}$. Denote the mean value of the solution $h(t)=\widehat{u_{0}}(t)=\frac{1}{2 \pi} \int_{\Omega} u(t, x) d x$. Then we represent $u(t, x)=h(t)+r(t, x)$. For $h(t)$ we obtain from Eq. (1.1)

$$
h^{\prime \prime}+2 \alpha h^{\prime}=\widehat{\mathcal{N}_{0}}(h+r), h(0)=\widehat{\phi_{0}}, h^{\prime}(0)=\widehat{\psi_{0}},
$$

where $\widehat{\mathcal{N}}_{0}(u)=-\frac{\lambda}{2 \pi} \int_{\Omega}|u(t, x)|^{\sigma} u(t, x) d x$. For the function $r(t, x)$ we can write the integral equation

$$
\begin{aligned}
r(t)= & \widetilde{\mathcal{G}}(t)\left(\phi-\widehat{\phi_{0}}\right)+\mathcal{G}(t)\left(\psi-\widehat{\psi_{0}}\right) \\
& +\int_{0}^{t} \mathcal{G}(t-\tau)\left(\mathcal{N}(u)(\tau)-\widehat{\mathcal{N}_{0}}(u)(\tau)\right) d \tau .
\end{aligned}
$$

Note that by (3.1) a rough estimate follows $|h(t)|+\left|h^{\prime}(t)\right| \leq \varepsilon$ for all $t \geq 0$. Let us prove the following estimate

$$
\|r(t)\|_{\dot{\mathbf{H}}^{1}}<C \varepsilon e^{-\Lambda t}
$$

for all $t \geq 0$, where $0<\Lambda<\mu, \mu=\operatorname{Re}\left(\alpha-\sqrt{\alpha^{2}-\beta}\right)>0$. We prove estimate (3.4) by the contradiction. Suppose that (3.4) is violated at some time $T_{1}$. Then by the continuity we have

$$
\|r(t)\|_{\dot{\mathbf{H}}^{1}} \leq C \varepsilon e^{-\Lambda t}
$$

for all $t \in\left[0, T_{1}\right]$. Note that $\widehat{\mathcal{N}(h)}{ }_{n}=-\frac{\lambda}{2 \pi} \int_{\Omega} e^{-i n x}|h|^{\sigma} h d x=0$ for all $n \neq 0$. Hence, by (3.1) and ( 3.5) we find the estimate

$$
\begin{aligned}
\|\mathcal{N}(u)(t)\|_{\dot{\mathbf{H}}^{0}}^{2} & =\sum_{|n| \geq 1}\left|\widehat{\mathcal{N}(u)_{n}}\right|^{2}=\|\mathcal{N}(u)-\mathcal{N}(h)\|_{\dot{\mathbf{H}}^{0}}^{2} \\
& \leq\|\mathcal{N}(u)-\mathcal{N}(h)\|_{\mathbf{H}^{0}}^{2}=\|\mathcal{N}(u)-\mathcal{N}(h)\|_{\mathbf{L}^{2}}^{2} \\
& \leq C\left(\|u\|_{\mathbf{L}^{\infty}}^{2 \sigma}+|h|^{2 \sigma}\right)\|r\|_{\mathbf{L}^{2}}^{2} \leq C \varepsilon^{2+2 \sigma} e^{-2 \Lambda t}
\end{aligned}
$$

for all $t \in\left[0, T_{1}\right]$. Then by (3.3) and estimates of Lemma 2.1 we find

$$
\begin{aligned}
\|r(t)\|_{\dot{\mathbf{H}}^{1}} & \leq C \varepsilon e^{-\mu t}+C \int_{0}^{t} e^{-\mu(t-\tau)}\|\mathcal{N}(u)(t)\|_{\dot{\mathbf{H}}^{0}} d \tau \\
& \leq C \varepsilon e^{-\mu t}+C \varepsilon^{1+\sigma} e^{-\mu t} \int_{0}^{t} e^{(\mu-\Lambda) \tau} d \tau<C \varepsilon e^{-\Lambda t}
\end{aligned}
$$


since $\varepsilon>0$ is sufficiently small. Thus, estimate (3.4) is true for all $t \geq 0$. Let us now consider the evolution of the mean value of the solution $h(t)$. By (3.2) we get

$$
h^{\prime \prime}+2 \alpha h^{\prime}=-\lambda|h|^{\sigma} h+\varphi(t),
$$

where

$$
\varphi(t)=\lambda\left(|h|^{\sigma} h-\frac{1}{2 \pi} \int_{\Omega}|u(t, x)|^{\sigma} u(t, x) d x\right)
$$

We have

$$
\begin{aligned}
|\varphi(t)| & =\left.|\lambda|\left|\frac{1}{2 \pi} \int_{\Omega}\left(|u(t, x)|^{\sigma} u(t, x)\right) d x-\right| h(t)\right|^{\sigma} h(t) \mid \\
& \leq C\left(\|u\|_{\mathbf{L}^{\infty}}^{\sigma}+|h|^{\sigma}\right)\|r(t)\|_{\mathbf{L}^{\infty}} \leq C \varepsilon^{1+\sigma} e^{-\Lambda t} .
\end{aligned}
$$

Therefore, by Lemma 2.4 we get the estimate

$$
\langle t\rangle^{\frac{1}{\sigma}}|h(t)|+\langle t\rangle^{\frac{1}{\sigma}+\frac{1}{2}}\left|h^{\prime}(t)\right| \leq C .
$$

This estimate along with inequality (3.4) yield the estimate (1.2) for the solution. Theorem 1.1 is proved.

\section{Proof of Theorem 1.2}

Now as in the previous section by (3.3), (3.6) and estimates of Lemma 2.1 we find

$$
\begin{aligned}
\|r(t)\|_{\dot{\mathbf{H}}^{1}} & \leq C \varepsilon^{1+\frac{\sigma}{2}} e^{-\mu t}+C \int_{0}^{t} e^{-\mu(t-\tau)}\|\mathcal{N}(u)(t)\|_{\dot{\mathbf{H}}^{0}} d \tau \\
& \leq C \varepsilon^{1+\frac{\sigma}{2}} e^{-\mu t}+C \varepsilon^{1+\sigma} e^{-\mu t} \int_{0}^{t} e^{(\mu-\Lambda) \tau} d \tau \leq C \varepsilon^{1+\frac{\sigma}{2}} e^{-\Lambda t}
\end{aligned}
$$

since $\varepsilon>0$ is sufficiently small. Thus, estimate

$$
\|r(t)\|_{\dot{\mathbf{H}}^{1}} \leq C \varepsilon^{1+\frac{\sigma}{2}} e^{-\Lambda t}
$$

is true for all $t \geq 0$. Also we can apply Lemma 2.5 to get the estimate

$$
|h(t)| \leq C \varepsilon\langle t\rangle^{\frac{1}{\sigma}},\left|h^{\prime}(t)\right| \leq C \varepsilon^{1+\frac{\sigma}{2}}\langle t\rangle^{-\frac{1}{\sigma}-\frac{1}{2}} .
$$

We now put $z=h^{\prime}+2 \alpha h$, then we can rewrite Eq. (3.7) in the form

$$
\frac{d z}{d t}=-\theta|z|^{\sigma} z(1-f(t))
$$

where $\theta=\lambda(2 \alpha)^{-1-\sigma}$,

$$
f(t)=\frac{\Phi(t)}{|z(t)|^{\sigma} z(t)}
$$

and

$$
\Phi(t)=|z|^{\sigma} z-\frac{(2 \alpha)^{1+\sigma}}{2 \pi} \int_{\Omega}|u(t, x)|^{\sigma} u(t, x) d x
$$


By the condition of Theorem 1.2 we have $z(0)=2 \alpha \varepsilon-O\left(\varepsilon^{1+\frac{\sigma}{2}}\right)>0$. Note that by virtue of estimate (4.2) we have

$$
\begin{aligned}
|\Phi(t)|= & \left.|\lambda|\left|\frac{(2 \alpha)^{1+\sigma}}{2 \pi} \int_{\Omega}\left(|u(t, x)|^{\sigma} u(t, x)\right) d x-\right| z\right|^{\sigma} z \mid \\
\leq & \left.|\lambda|\left|\frac{(2 \alpha)^{1+\sigma}}{2 \pi} \int_{\Omega}\left(|u(t, x)|^{\sigma} u(t, x)\right) d x-\right| h(t)\right|^{\sigma} h(t) \mid \\
& +\left.|\lambda|\left|(2 \alpha)^{1+\sigma}\right| h(t)\right|^{\sigma} h(t)-|z|^{\sigma} z \mid \\
\leq & C \varepsilon^{\sigma}\|r(t)\|_{\dot{\mathbf{H}}^{1}}+C\left(|h|^{\sigma}+|z|^{\sigma}\right)\left|h^{\prime}\right| \\
\leq & C \varepsilon^{1+\frac{3}{2} \sigma} e^{-\Lambda t}+C \varepsilon^{1+2} \leq C \varepsilon^{1+\frac{3}{2} \sigma}\langle t\rangle^{-\frac{1}{\sigma}-\frac{3}{2}} .
\end{aligned}
$$

We integrate (4.3) with respect to time

$$
z(t)=z(0)\left(1+\sigma \theta|z(0)|^{\sigma}\left(t-\int_{0}^{t} f(\tau) d \tau\right)\right)^{-\frac{1}{\sigma}}
$$

Let us prove the estimate

$$
|f(t)|<\frac{1}{2}\langle t\rangle^{-\frac{1}{2}}
$$

for all $t \geq 0$. We note by (4.4) that $|f(0)|=\frac{|\Phi(0)|}{|z(0)|^{1+\sigma}} \leq C \varepsilon^{\frac{\sigma}{2}}$. By the continuity in time we can find a maximal time interval $[0, T]$ such that the estimate is true

$$
|f(t)| \leq \frac{1}{2}\langle t\rangle^{-\frac{1}{2}}
$$

for all $t \in[0, T]$. Hence,

$$
|z(t)|^{1+\sigma} \geq|z(0)|^{1+\sigma}\left(1+\frac{\sigma \theta}{2}|z(0)|^{\sigma} t\right)^{-1-\frac{1}{\sigma}}
$$

and by virtue of (4.4) we have

$$
\begin{aligned}
|f(t)| & \leq \frac{|\Phi(t)|}{|z(0)|^{1+\sigma}}\left(1+\frac{\sigma \theta}{2}|z(0)|^{\sigma} t\right)^{1+\frac{1}{\sigma}} \\
& \leq C \varepsilon^{\frac{\sigma}{2}}\langle t\rangle^{-\frac{1}{\sigma}-\frac{3}{2}}\left(1+\frac{\sigma \theta}{2} \varepsilon^{\sigma} t\right)^{1+\frac{1}{\sigma}}<\frac{1}{2}\langle t\rangle^{-\frac{1}{2}}
\end{aligned}
$$

for all $t \in[0, T]$, since $\varepsilon>0$ is sufficiently small. Thus, estimate (4.6) is true for all $t \geq 0$. By Eqs. (4.6) and (4.5) we get the asymptotic formula

$$
z(t)=(\sigma \theta)^{-\frac{1}{\sigma}} t^{-\frac{1}{\sigma}}+O\left(t^{-\frac{1}{\sigma}-\frac{1}{2}}\right) .
$$

By virtue of (4.1) and (4.2) we have the estimate

$$
\left|u(t, x)-\frac{z(t)}{2 \alpha}\right| \leq|r(t, x)|+\left|\frac{h^{\prime}(t)}{2 \alpha}\right| \leq C t^{-\frac{1}{\sigma}-\frac{1}{2}}
$$


Thus, asymptotics (1.3) follows. Theorem 1.2 is proved.

Remark 4.1. By the proof of Theorem 1.2 we can see that if we can guarantee that the mean value $h(t) \equiv 0$ for all $t \geq 0$, then the asymptotics is more rapid

$$
u(t, x)=r(t, x)=O\left(e^{-\Lambda t}\right)
$$

as $t \rightarrow \infty$ uniformly with respect to $x \in \Omega$. For example, if the initial data $\phi$ and $\psi$ are odd functions, then it is easy to see that $u(t, x)$ is odd for all $t \geq 0$. Hence, in this case we have $h(t) \equiv 0$ for all $t \geq 0$.

\section{Acknowledgments}

The work of P.I.N. and J.A.R.C. is partially supported by CONACYT and the work of N.H. is partially supported by KAKENHI (no. 19340030).

\section{References}

[1] Biler, P.: Large-time behaviour of periodic solutions to dissipative equations of Korteweg-de Vries-Burgers type. Bull. Pol. Acad. Sci. Math. 32, 401-405 (1984)

[2] Bu, C., Shull, R., Zhao, K.: A periodic boundary value problem for a generalized 2D Ginzburg-Landau equation. Hokkaido Math. J. 27(1), 197-211 (1998)

[3] Constantin, A., Escher, J.: Well-posedness, global existence, and blowup phenomena for a periodic quasi-linear hyperbolic equation. Commun. Pure Appl. Math. 51(5), 475-504 (1998)

[4] Dafermos, C.M.: Large time behavior of periodic solutions of hyperbolic systems of conservation laws. J. Differ. Equ. 121(1), 183-202 (1995)

[5] Doering, C.R., Gibbon, J.D., Levermore, C.D.: Weak and strong solutions of the complex Ginzburg-Landau equation. Physica D 71(3), 285-318 (1994)

[6] Duan, J., Holmes, P., Titi, E.S.: Global existence theory for a generalized Ginzburg-Landau equation. Nonlinearity 5(6), 1303-1314 (1992)

[7] Fife, P.C.: Asymptotic states for equations of reaction and diffusion. Bull. Am. Math. Soc. 84, 693-726 (1978)

[8] Foias, C., Kukavica, I.: Determining nodes for the Kuramoto-Sivashinsky equation. J. Differ. Equ. 7(2), 365-373 (1995)

[9] Georgiev, V., Todorova, G.: Existence of a solution of the wave equation with nonlinear damping and source terms. J. Differ. Equ. 109(2), 295-308 (1994)

[10] Georgiev, V., Milani, A.: On the asymptotic behavior of semilinear wave equations with degenerate dissipation and source terms. Nonlinear Differ. Equ. Appl. 5(1), 53-68 (1998)

[11] Guo, B., Xiang, X.M.: The large time convergence of spectral method for generalized Kuramoto-Sivashinsky equations. J. Comput. Math. 15(1), 1-13 (1997) 
[12] Hayashi, N., Kaikina, E.I., Naumkin, P.I.: Damped wave equation with a critical nonlinearity. Trans. Am. Math. Soc. 358(3), 1165-1185 (2006)

[13] Hayashi, N., Kaikina, E.I., Naumkin, P.I.: Damped wave equation with supercritical nonlinearities. Differ. Integral Equ. 17(5-6), 637-652 (2004)

[14] Hayashi, N., Kaikina, E.I., Naumkin, P.I.: Damped wave equation in the subcritical case. J. Differ. Equ. 207(1), 161-194 (2004)

[15] Hayes, B.T.: Stability of solutions to a destabilized Hopf equation. Commun. Pure Appl. Math. 48(2), 157-166 (1995)

[16] Huang, G., Russell, D.L.: Asymptotic properties of solutions of a KdV-Burgers equation with localized dissipation. J. Math. Syst. Estim. Control 8(4), 467$470(1998)$

[17] Kaikina, E.I., Naumkin, P.I., Shishmarev, I.A.: Periodic problem for a model nonlinear evolution equation. Adv. Differ. Equ. 7(5), 581-616 (2002)

[18] Karch, G.: Self-similar large time behavior of solutions to Korteweg-de Vries-Burgers equation. Nonlinear Anal. Theory Methods Appl. 35A(2), 199219 (1999)

[19] Kirsch, W., Kutzelnigg, A.: Time asymptotics for solutions of the Burgers equation with a periodic force. Math. Z. 232(4), 691-705 (1999)

[20] Li, T.T., Zhou, Y.: Breakdown of solutions to $\square u+u_{t}=|u|^{1+\alpha}$. Discrete Conti. Dyn. Syst. 1(4), 503-520 (1995)

[21] Lu, D., Tian, L., Liu, Z.: Wavelet basis analysis in perturbed periodic KdV equation. Appl. Math. Mech. Engl. Ed. 19(11), 1053-1058 (1998)

[22] Marcati, P.: Decay and stability for nonlinear hyperbolic equations. J. Differ. Equ. 55(1), 30-58 (1984)

[23] Marcati, P., Nishihara, K.: The $\mathbf{L}^{p}-\mathbf{L}^{q}$ estimates of solutions to one-dimensional damped wave equations and their application to the compressible flow through porous media. J. Differ. Equ. 191(2), 445-469 (2003)

[24] Matsumura, A.: On the asymptotic behavior of solutions of semi-linear wave equations. Publ. Res. Inst. Math. Sci. Kyoto Univ. 12, 169-189 (1976)

[25] Nishida, T.: Nonlinear hyperbolic equations and related topics in fluid dynamics. Publications Mathématiques d'Orsay, No. 78-02. Département de Mathématique, Université de Paris-Sud, Orsay, 1978. iv+123 pp

[26] Nishihara, K.: $\mathbf{L}^{p}-\mathbf{L}^{q}$ estimates of solutions to the damped wave equation in 3-dimensional space and their application. Math. Z 244, 631-649 (2003)

[27] Nishihara, K., Zhao, H.: Decay properties of solutions to the Cauchy problem for the damped wave equation with absorption. J. Math. Anal. Appl. 313(2), 598610 (2006)

[28] Ono, K.: Global existence and asymptotic behavior of small solutions for semilinear dissipative wave equations. Discrete Conti. Dyn. Syst. 9, 651-662 (2003) 
[29] Promislov, K., Temam, R.: Localization and approximation of attractors for the Ginzburg-Landau equation. J. Dyn. Differ. Equ. 3(4), 491-514 (1991)

[30] Pucci, P., Serrin, J.: Precise damping conditions for global asymptotic stability for nonlinear second order systems. II. J. J. Differ. Equ. 113(2), 505-534 (1994)

[31] Pucci, P., Serrin, J.: Asymptotic stability for nonautonomous dissipative wave systems. Commun. Pure Appl. Math. 49(2), 177-216 (1996)

[32] Pucci, P., Serrin, J.: Local asymptotic stability for dissipative wave systems. Israel J. Math. 104, 29-50 (1998)

[33] Russell, D.L., Zhang, B.-Y.: Smoothing and decay properties of solutions of the Korteweg-de Vries equation on a periodic domain with point dissipation. J. Math. Anal. Appl. 190(2), 449-488 (1995)

[34] Sinestrari, C.: Large time behaviour of solutions of balance laws with periodic initial data. Nonlinear Differ. Equ. Appl. 2(1), 111-131 (1995)

[35] Todorova, G., Yordanov, B.: Critical exponent for a nonlinear wave equation with damping. J. Differ. Equ. 174, 464-489 (2001)

[36] Varlamov, V.V.: On spatially periodic solutions of the damped Boussinesq equation. Differ. Integral Equ. 10(6), 1197-1211 (1997)

[37] Xing, J.: Global strong solution for a class of Burgers-BBM type equation. Appl. Math. J. Chin. Univ. 6(1), 31-37 (1991)

[38] Yang, Y.: Global spatially periodic solutions to the Ginzburg-Landau equation. Proc. R. Soc. Edinb. Sect. A 110(3/4), 263-273 (1988)

[39] You, Y.: Global dynamics of dissipative generalized Korteweg-de Vries equations. Chin. Ann. Math. Ser. B 17(4), 389-402 (1996)

[40] Zuazua, E.: Some recent results on the large time behavior for scalar parabolic conservation laws, in Elliptic and parabolic poblems. Proc. 2nd European Conference. Pitman Res. Notes Math. Ser. 325, pp. 251-263 (1995)

\author{
N. Hayashi \\ Department of Mathematics, \\ Graduate School of Science, \\ Osaka University, \\ Osaka, Toyonaka 560-0043, \\ Japan \\ e-mail: nhayashi@math.sci.osaka-u.ac.jp
}


P. I. Naumkin, J. A. Rodriguez-Ceballos

Instituto de Matemáticas,

Universidad Nacional Autónoma de México,

Campus Morelia, AP 61-3 (Xangari),

Morelia 58089, Michoacán,

Mexico

e-mail: pavelni@matmor.unam.mx

J. A. Rodriguez-Ceballos

e-mail: jarturo@matmor.unam.mx

Received: 16 March 2009.

Accepted: 18 January 2010. 